\title{
Diminishing Risk-Taking Behavior by Modulating Activity in the Prefrontal Cortex: A Direct Current Stimulation Study
}

\author{
Shirley Fecteau, ${ }^{1}$ Daria Knoch,,${ }^{2,34}$ Felipe Fregni, ${ }^{1}$ Natasha Sultani, ${ }^{5}$ Paulo Boggio, ${ }^{5}$ and Alvaro Pascual-Leone ${ }^{1,6}$ \\ ${ }^{1}$ Berenson-Allen Center for Noninvasive Brain Stimulation, Harvard Medical School and Beth Israel Deaconess Medical Center, Boston, Massachusetts \\ 02215, ${ }^{2}$ Institute for Empirical Research in Economics, University of Zurich, 8006 Zurich, Switzerland, ${ }^{3}$ Department of Neurology, University Hospital \\ Zurich, 8091 Zurich, Switzerland, ${ }^{4}$ Collegium Helveticum, 8092 Zurich, Switzerland, ${ }^{5}$ Núcleo de Neurociências, Centro de Ciências Biológicas e da Saúde, \\ Universidade Presbiteriana Mackenzie, 01302-907, Sao Paulo, Brazil, and 'Institut Guttmann de Neurorehabilitación, 08027 Barcelona, Spain
}

Studies have shown increased risk taking in healthy individuals after low-frequency repetitive transcranial magnetic stimulation, known to transiently suppress cortical excitability, over the right dorsolateral prefrontal cortex (DLPFC). It appears, therefore, plausible that differential modulation of DLPFC activity, increasing the right while decreasing the left, might lead to decreased risk taking, which could hold clinical relevance as excessively risky decision making is observed in clinical populations leading to deleterious consequences. The goal of the present study was to investigate whether risk-taking behaviors could be decreased using concurrent anodal transcranial direct current stimulation (tDCS) of the right DLPFC, which allows upregulation of brain activity, with cathodal tDCS of the left DLPCF, which downregulates activity. Thirty-six healthy volunteers performed the risk task while they received either anodal over the right with cathodal over the left DLPFC, anodal over the left with cathodal over the right DLPFC, or sham stimulation. We hypothesized that right anodal/left cathodal would decrease risk-taking behavior compared with left anodal/right cathodal or sham stimulation. As predicted, during right anodal/left cathodal stimulation over the DLPFC, participants chose more often the safe prospect compared with the other groups. Moreover, these participants appeared to be insensitive to the reward associated with the prospects. These findings support the notion that the interhemispheric balance of activity across the DLPFCs is critical in decision-making behaviors. Most importantly, the observed suppression of risky behaviors suggests that populations with boundless risk-taking behaviors leading to negative real-life consequences, such as individuals with addiction, might benefit from such neuromodulation-based approaches.

Key words: tDCS; decision making; dorsolateral prefrontal cortex; inhibitory control; laterality; risk-taking behavior

\section{Introduction}

Recent evidence from repetitive transcranial magnetic stimulation (rTMS) studies demonstrates a causal role of the right lateral prefrontal cortex in human decision making. Suppression of activity in the dorsolateral prefrontal cortex (DLPFC) using lowfrequency rTMS alters decision making related to fairness (van't Wout et al., 2005; Knoch et al., 2006a) and increases the choice of high-risk prospects (Knoch et al., 2006b). These neurostimulation studies found that right-sided but not left-sided rTMS has an impact on individual and social decision making. For instance, subjects displayed significantly riskier decision making, choosing a larger potential reward even at the greater risk of penalty after disruption of the right, but not the left, DLPFC (Knoch et al., 2006a). These findings have led to the notion that the right

Received March 8, 2007; revised Aug. 8, 2007; accepted Aug. 13, 2007.

This work was supported by a fellowship from the Fonds de Recherche en Santé du Québec and the Canadian Institutes of Health Research to S.F. and by National Institutes of Health Grants K24 RR018875, R01-MH069898, R01-DC05672, R01-NS47754, R01-NS20068, R01-EY12091, R01-EB005047, and R03-DK071851, and the HarvardThorndike General Clinical Research Center (National Center for Research Resources M01 RR01032) to A.P.-L.

Correspondence should be addressed to Dr. Alvaro Pascual-Leone, Berenson-Allen Center for Noninvasive Brain Stimulation, Department of Neurology, Beth Israel Deaconess Medical Center, 330 Brookline Avenue, KS 452, Boston, MA 02215. E-mail: apleone@bidmc.harvard.edu.

D01:10.1523/JNEUROSCI.3283-07.2007

Copyright $\odot 2007$ Society for Neuroscience $\quad$ 0270-6474/07/2712500-06\$15.00/0
DLPFC plays a crucial role in the suppressive control of superficially seductive options. Disruption of the DLPFC appears to decrease such inhibitory control, leading to increased risk-taking behavior.

A follow-up question with potential clinical significance is whether increasing the level of activity in the right DLPFC might promote the inhibitory control of prepotent, impulsive responses and therefore diminish risk-taking behavior. If so, such an intervention might prove useful in clinical populations with right prefrontal syndromes, such as drug or nonsubstance addictions (e.g., pathological gambling), in which impairments of decision making seem to reflect a breakdown of these control processes (Starkstein and Robinson, 1997). Preliminary findings in cocaine addicts reveals that high-frequency rTMS to the right DLPFC, which is thought to increase cortical excitability in the targeted brain region (Gangitano et al., 2002), reduces craving (Camprodon et al., 2007).

It is important to recognize that when targeting one DLPFC, noninvasive brain stimulation induces changes locally but also at distant sites along the involved neural network. Critically, this includes interhemispheric effects modifying activity in the homologous regions of the unstimulated hemisphere as demonstrated by studies in animal models and neuroimaging studies in humans (Wagner et al., 2007). Therefore, even when stimulation is applied unilaterally, there is likely some component of modu- 

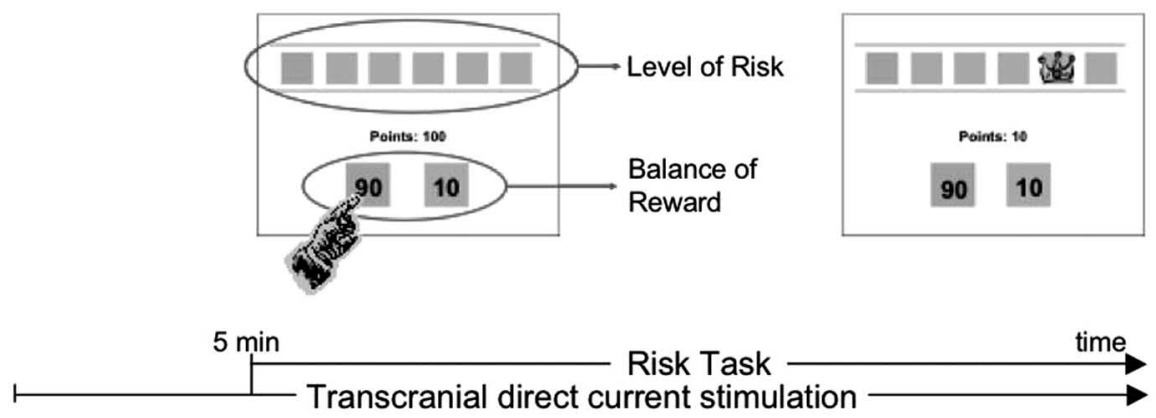

Figure 1. Schematic representation of the experimental design. Each participant started to perform the risk task after receiving 5 min of stimulation. Stimulation continued throughout the task.

lation of activity in the contralateral hemisphere that may, in fact, contribute to the observed behavioral effects. Previous findings on the behavioral changes in risk taking after stimulation using rTMS over the right DLPFC (Knoch et al., 2006a,b) may, in fact, be attributable to an inhibition of activity of the right DLPFC, coupled with an increase in activity in the homologous, contralateral region (left DLPFC). Indeed, we have shown that bilateral neuromodulation of the DLPFC can lead to behavioral changes in risk taking under ambiguity, whereas no significant behavioral change was observed with unilateral neuromodulation of the DLPFC (Fecteau et al., 2007).

The aim of the present study was to investigate whether risk taking in normal volunteers can be reduced with transcranial direct current stimulation (tDCS) modulating activity in the right and left DLPFC in opposite directions simultaneously. We used a well known gambling paradigm, the risk task, that provides a measure of decision making under risk (Rogers et al., 1999) and applied anodal tDCS to the right or the left prefrontal cortex, coupled with cathodal tDCS over the contralateral area. Anodal tDCS is thought to increase excitability in the targeted brain region and induces a stronger modulatory effect on cortical excitability than rTMS, as shown by studies evaluating the impact of these two techniques on motor cortex excitability (Nitsche and Paulus, 2001; Romero et al., 2002), whereas cathodal tDCS is known to decrease cortical excitability (Ardolino et al., 2005). tDCS has the additional advantages of allowing testing of behaviors during stimulation without nonspecific disruptions of task performance and providing for a reliable sham condition (Gandiga et al., 2006). The cortical excitability shifts during tDCS are believed to be attributable to subthreshold neuronal membrane depolarization (Nitsche and Paulus, 2000; Liebetanz et al., 2002; Priori, 2003). The resting membrane modulation appears to be caused by the electrical current flow, which involves opening or closing of voltage-gated ion channels (Purpura and McMurtry, 1965; Nitsche et al., 2003). tDCS induces modulation of excitability, which can transiently change behavioral performance in healthy humans (Nitsche et al., 2003b; Fregni et al., 2005; Iyer et al., 2005; Vines et al., 2006) and neurological patients (Hummel et al., 2005; Fregni et al., 2006).

\section{Materials and Methods}

Participants. Thirty-six healthy college students (11 men; mean age, $20.3 \pm 1.7$ years; three left-handed as assessed by the Oldfield Questionnaire) performed the risk task while receiving anodal tDCS to either the right or the left DLPFC, coupled with cathodal tDCS to the contralateral DLPFC (referred to as right anodal/left cathodal and left anodal/right cathodal) or sham tDCS. Participants were on no chronic or acute medications, had no history of neurological or psychiatric diseases, and had normal physical and neurological examinations. All were naive to tDCS, the risk task, and the nature of the experiment and were not informed about the experimental variable tested. Participants gave informed written consent before entering the study, which was approved by the local ethics committee. The study was performed at Mackenzie University (Sao Paulo, Brazil).

Risk task. The risk task (Rogers et al., 1999) is a decision-making task involving gambling. This task provides a measure of decision making under risk with little requirements on strategy and working memory. In each of the 100 trials, participants were presented with six horizontally arranged boxes that could be pink or blue. The ratio of pink and blue boxes varied from trial to trial and could be $5: 1,4: 2$, or $3: 3$. Participants had to pick the color of the box that hid the winning token. They were told that the token was equally likely to be hidden in any of the boxes. Therefore, for each trial, the ratio of pink-to-blue boxes (referred to as level of risk) effectively determined the probability of finding that winning token and thus the level of risk of the choice. Participants were rewarded with points for correctly guessing the color of the box hiding the winning token and punished by losing points for picking the incorrect color. The amount of reward (or penalty) points associated with any scenario (e.g., five blue and one pink box) varied (90:10, 80:20, 70:30, 60:40; referred to as balance of reward) and was clearly indicated on the screen. Importantly, the conflict inherent in risk taking was reflected by the fact that the largest reward was always associated with the least likely of the two outcomes (i.e., the most risky option). Thus, in a trial with five blue boxes and one pink box, the winning token was much more likely to be hidden in a blue box (five-insix probability) than in the one pink box (one-in-six probability). In this case, if the participant picked pink and was correct, he would be awarded the number of points associated with the choice of pink (which would be higher than the points associated with the choice of blue). Conversely, picking pink and missing the winning token would result in a loss of the same, larger number of points. Participants' aim was to earn as many points as possible.

$t D C S$. Direct current was induced by two saline-soaked surface sponge electrodes $\left(35 \mathrm{~cm}^{2}\right)$ and delivered by a battery-driven, constant current stimulator. The device used, developed by our group, is particularly reliable for double-blind studies: a switch can be activated to interrupt the electrical current while maintaining the ON display and showing the stimulation parameters throughout the procedure to the experimenter and participant. For technical details, contact P.B. at boggio@usp.br.

Participants were randomly assigned to receive left anodal/right cathodal tDCS $(n=12)$, right anodal/left cathodal tDCS $(n=12)$, or sham stimulation $(n=12)$. For stimulation left anodal/right cathodal, the anode electrode was placed over the left F3 (EEG 10/20 system) and the cathode electrode was placed over the right F4. For stimulation right anodal/left cathodal, the polarity was reversed: the anode electrode was placed over F4 (international EEG 10/20 system) and the cathode electrode was placed over F3. For active stimulation, participants received a constant current of $2 \mathrm{~mA}$ intensity. tDCS started 5 min before the task began and was delivered during the entire course of the risk task, which lasted $<10 \mathrm{~min}$ (Fig. 1). For sham stimulation, the electrodes were placed at the same positions as for active stimulation (F3 and F4), but the stimulator was turned on only for $30 \mathrm{~s}$. Thus, participants felt the initial itching sensation associated with $\mathrm{tDCS}$ but received no active current for the rest of the stimulation period. This method of sham stimulation has been shown to be reliable (Gandiga et al., 2006).

Data analysis. The outcome measures in the present study were as follows: (1) the choice of low risk (i.e., safe prospect) versus high risk in each trial (binary variable). This measure is the percentage of instances in which participants chose the high-probability option (i.e., the choice of low-risk; the color corresponding to more boxes); and (2) the decision time (i.e., how long it took the participants to decide which color of box was hiding the winning token; measured in milliseconds) (continuous variable). 
Performance on all 100 trials of the task (excluding the neutral conditions; i.e., equal number of blue and pink boxes) was analyzed. The number of times participants choose the most likely outcome and the decision time during left anodal/right cathodal, right anodal/left cathodal, and sham stimulation was calculated and averaged. Outliers were defined as $2 \mathrm{SD}$ above or below individual mean of decision time for the decision time and 2 SD above or below the group mean for the total points earned. Analyses were performed using SAS (Cary, NC) statistical software (version 9.1). We used a mixed linear model to analyze decisiontime difference across the groups. We modeled decision-time change using the covariates of group (left anodal/right cathodal stimulation, right anodal/left cathodal stimulation, sham stimulation), balance of reward (90:10, 80:20, 70:30, 60:40), level of risk (low risk, high risk), and interaction terms group $\times$ balance of reward $\times$ level of risk. For the outcome considering the choice of low risk versus high risk (binary outcome), we performed a logistic regression model in which the dependent variable was the choice (low risk, high risk) and the independent variables were group (left anodal/right cathodal stimulation, right anodal/ left cathodal stimulation, sham stimulation), balance of reward (90:10, $80: 20,70: 30,60: 40)$, and interaction group $\times$ balance of reward. Statistical analysis applied Bonferroni adjustments for multiple comparisons. To allow a full assessment of the findings, we report threshold level for significance considering the multiple comparisons (corrected $p$ values) as well as uncorrected $p$ values given the relatively small sample size and the likelihood that Bonferroni correction may impose an excessively conservative adjustment.

\section{Results}

None of the healthy volunteers experienced adverse effects during or after tDCS. Participants perceived a slight itching sensation under the electrodes during approximately the first $30 \mathrm{~s}$ of stimulation. Subjects in the sham stimulation group were truly blinded to the nature of stimulation, reported the same initial itching sensation, and when explicitly asked, believed to have undergone real stimulation. The duration to perform the risk task was similar for the three groups of subjects $(<15 \mathrm{~min}$, regardless of the stimulation condition; ANOVA, $p=0.461)$.

We first tested our main a priori hypothesis based on previous findings by Knoch et al. (2006a), which postulated that participants receiving anodal tDCS to the right DLPFC coupled with cathodal tDCS to the left DLPFC would display risk-averse behavior on the risk task. To do so, we used a specific test model using choice as the dependent variable. Results revealed a main effect of group $(p<0.0001)$ : participants with right anodal/left cathodal stimulation chose more often low-risk prospects compared with participants with sham stimulation [odds ratio (OR), 2.5; 95\% confidence interval (CI), 1.8-3.3] and those with left anodal/right cathodal (OR, 2.8; 95\% CI, 2.1-3.8). Moreover, there was no difference between groups receiving left anodal/ right cathodal and sham stimulation (OR, 0.88; 95\% CI, 0.7-1.1) (Fig. 2). Also, there was no significant difference between women and men in their choices (unpaired $t$ test, $p=0.46$ ).

We then tested whether the balance of reward influenced the choice of the low-risk prospect, as reported previously (Rogers et al., 1999; Knoch et al., 2006a). Results revealed a significant main effect of balance of reward ( $p=0.0019)$ : participants tended to choose less often the low-risk prospect, compared with the risky one, when its associated reward was diminished. We then investigated whether the difference in regard to the balance of reward was similar across groups and found a significant interaction between group $\times$ balance of reward $(p<0.0001)$. Participants with right anodal/left cathodal choose more often the safe prospect, regardless of its associated reward, compared with participants with sham stimulation [reward 90:10, $p<0.01$; reward 80:20, $p<0.01$; reward 70:30, $p<0.001$; reward 60:40, $p<0.01$; significance threshold for the Bonferroni

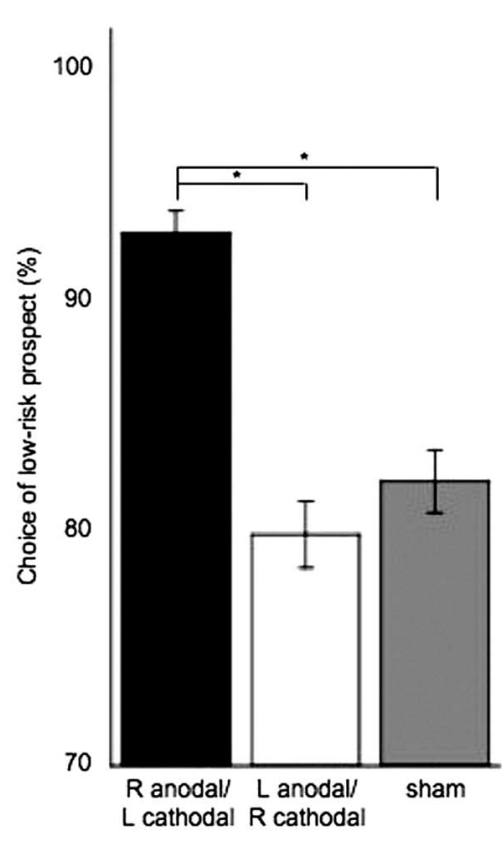

Figure 2. Choice between low-risk and high-risk prospect (in percentages; SEM). Choice of low-risk prospect was significantly different across groups. Participants receiving right anodal/ left cathodal DLPFC stimulation choose more often the low-risk prospect (92\%) compared with those with left anodal/right cathodal DLPFC stimulation (79\%) and sham stimulation (82\%). Asterisks indicate significant difference between the two groups of comparison using a corrected $\alpha$ value. R, Right; L, left.

correction (corrected $p$ value) was $<0.0125]$. Participants with right anodal/left cathodal appeared to be so conservative in their choice that that they were not even influenced by the reward. Finally, there was a significant interaction effect of group $\times$ level of risk $\times$ balance of reward $(p<0.0001)$, suggesting that tDCS was also an effect modifier for the level of risk $(4: 2,5: 1)$.

In regard to the total points earned, participants receiving right anodal/left cathodal earned significantly more points compared with those receiving left anodal/right cathodal $[p=0.0468$; significance threshold for the Bonferroni correction (corrected $p$ value) was $<0.025]$, but not compared with sham stimulation $(p=0.1121)$. There was no difference between the total points earned between participants with left anodal/right cathodal compared with sham stimulation ( $p=0.4257$ ) (Fig. 3).

We also tested whether the decision times were longer when participants were confronted to a 4:2 versus a 5:1 scenario, as found by Rogers et al. (1999) and Knoch et al. (2006a). There was a main effect of level of risk $(F=11.87 ; p=0.001)$. Participants decided slower when confronted with the safer scenario $(4: 2$ choice) compared with the higher-risk scenario (5:1 choice). There was no interaction group $\times$ level of risk $(p=0.18)$. However, there was a significant difference across the groups. Compared with sham stimulation, participants with right anodal/left cathodal stimulation were significantly faster $(p<0.0001)$ and those with left anodal/right cathodal stimulation were significantly slower $(p<0.0029)$. Furthermore, there was a significant interaction across group $\times$ balance of reward $[p<0.0001$; significance threshold for the Bonferroni correction (corrected $p$ value) was $<0.0125]$, suggesting that differences in decision time between groups varied according to the reward. We also found a significant interaction between group $\times$ level of risk $\times$ balance of reward $(p<0.0001)$. Finally, there was no significant gender difference in terms of decision time (ANOVA, $p=0.689$ ). 


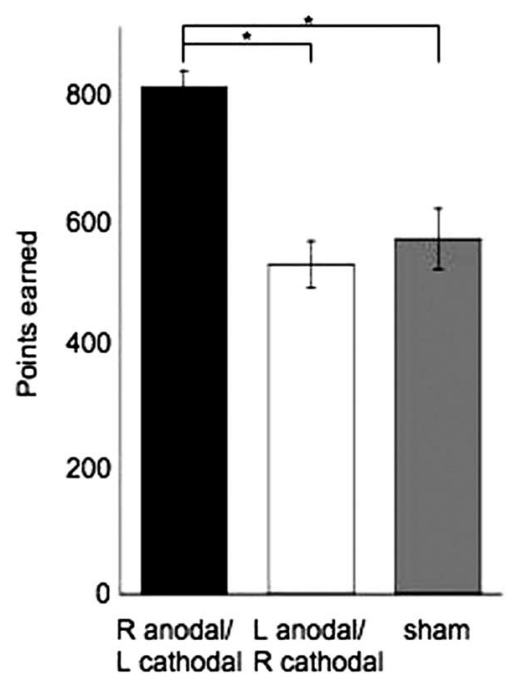

Figure 3. Total points earned (mean and SEM). Participants with right anodal/left cathodal DLPFC stimulation earned more points $(803 \pm 27)$ than those with left anodal/right cathodal DLPFC stimulation (523 \pm 37 ) and with sham stimulation (563 \pm 48 ). Asterisks indicate significance using the uncorrected $p$ value. $R$, Right; L, left.

\section{Discussion}

In the present study, we found that modulation of activity in the DLPFC using tDCS can influence decision-making behaviors in healthy individuals, supporting previous work showing that rTMS over the DLPFC has an impact on decision-making behaviors (van't Wout et al., 2005; Knoch et al., 2006a,b). Overall, when healthy participants were receiving anodal stimulation over the right DLPFC coupled with cathodal over the left DLPFC, they chose more often the safe prospects, were faster to choose between the low-risk and high-risk prospects, and earned more points compared with participants with sham stimulation. Conversely, subjects receiving anodal stimulation over the left DLPFC coupled with cathodal over the right DLPFC, did not differ in their choice related to risk-taking behavior from those receiving sham stimulation, but they were slower to make their decision. Previous work has shown that inhibiting cortical excitability in the right DLPFC with rTMS increases risk-taking behaviors. Here we showed that anodal stimulation, which is thought to externally upregulate cortical excitability, coupled with cathodal stimulation (known to decrease excitability), leads to the opposite decision-making behavior (i.e., a risk-averse response style). Interestingly, in a previous study, we observed a decrease in risk taking under ambiguity [Balloon Analog Risk Task (BART)] when anodal stimulation was applied to either the right or the left DLPFC, coupled with cathodal stimulation over the contralateral DLPFC (Fecteau et al., 2007). This difference between the two studies might be task related (risk task vs BART) but needs further investigation. Some decision-making tasks involve risk, whereas others involve ambiguity (Bechara et al., 2005), and a differential pattern of brain activity has been associated with these processes (Krain et al., 2006). In the task used in the present study, the subject is in a situation in which probabilities are known and the lower probability outcome is worth more than the higher probability outcome. On the contrary, the BART used in our previous study (Fecteau et al., 2007) involves ambiguity in which the probabilities are unknown.

As expected, participants were attracted to choose the safe prospect when the reward was smaller, supporting findings by Rogers et al. (1999) and Knoch et al. (2006a). Interestingly, participants with right anodal/left cathodal stimulation were significantly less influenced by the reward compared with those with sham stimulation, and that was observed for each balance of reward (90:10, 80:20, 70: $30,60: 40)$. One possible explanation is that anodal stimulation over the right DLPFC with cathodal stimulation over the left DLPFC suppressed the willingness to choose the high-risk prospect when the reward is greater. Risk-averse behavior induced by right anodal/left cathodal stimulation might have been so strong (they choose lowrisk prospect in $92 \%$ of the trials) that these participants were simply insensitive to reward. An alternative explanation is that the lack of influence related to the reward might reflect a floor effect. This result differs from findings by Knoch et al. (2006a), who reported that stimulation groups (low-frequency rTMS over the right or the left DLPFC, or sham rTMS) showed similar patterns of choice in relation to the reward. Several factors might have contributed to this difference between the two studies. First, tDCS might exert a different effect or modulate brain activity stronger than rTMS. Furthermore, in the study by Knoch et al. (2006a), participants performed the risk task after low-frequency rTMS, whereas in the present study, participants performed the risk task during stimulation, presumably during the peak of the stimulation effects.

Participants with right anodal/left cathodal stimulation over the DLPFC did not only adopt a strong risk-averse response style, they were also significantly faster to make their choice than those with sham stimulation. In contrast, participants with left anodal/ right cathodal stimulation, although they did not differ from those with sham stimulation in terms of how often they choose the safe or risky prospect, were much slower to make their choice. The change in response time is most unlikely to be caused by a task-independent effect of the tDCS on motor and response speed. Most participants (33 of the 36 participants) performed the task with their right hand, and increasing activity in the right hemisphere would have lead to slower speed response through transcallosal inhibition, whereas enhancing activity in the left one would have led to faster speed time if the response time was only an index of motor and response speed. In addition, response times in all stimulation groups were modulated by the level of risk (scenarios 5:1, 4:2) and the potential reward. Finally, all three groups of participants completed the risk task in a similar amount of time. It is possible that participants with right anodal/ left cathodal stimulation were so risk averse that they did not even seriously consider the choice of the risky prospects.

\section{Limitations}

The most important limitation of the present study is that we cannot derive whether the behavioral effects were caused by anodal tDCS, or by both anodal/cathodal tDCS. Neither the present nor the previous brain stimulation studies on the role of the DLPFC on risk-taking behavior (van't Wout et al., 2005; Knoch et al., 2006a,b) can conclusively establish whether the impact on decision making is solely attributable to the modulation of activity in the right DLPFC, or whether the behavioral effects are the result of changing the balance of activity across both DLPFCs. Modulating activity in one DLPFC is presumed to have transcallosal (and opposite) effects on the activity of its homolog in the contralateral (unstimulated) hemisphere. We believe that the behavioral changes were likely attributable to both anodal/cathodal tDCS modifying activity in right and left DLPFCs in opposite directions simultaneously. This conclusion is based on findings from a previous study (Fecteau et al., 2007). We showed that unilateral neuromodulation of the DLPFC (anodal over the right and cathodal over the contralateral supraorbital area) did not lead to changes in another decision-making task (the BART), 
whereas anodal over the right DLPFC coupled with cathodal over the left DLPFC resulted in behavioral modifications. Moreover, in the present study, cathodal tDCS over the right DLPFC coupled with anodal over the left DLPFC did not lead to an increase in risk taking, as would be suggested by the previous study by Knoch et al. (2006a). This indicates that cathodal tDCS appears to have less effect on behavioral changes than anodal tDCS, or, alternatively, the direction of the current is critical for the effects on this task. Cathodal tDCS might have a weaker impact on brain activity than inhibitory low-frequency rTMS. tDCS appears to be neuromodulatory intervention, and rTMS appears to be both a neuromodulatory and neurostimulatory intervention. The physiological impact of both techniques are therefore likely different and thus may lead to different behavioral effects. Future studies should include neuroimaging measures to explore which neural changes are associated with the neuromodulation leading to the behavioral effects and also explore other paradigms of stimulation such as that using unilateral stimulation (Nitsche et al., 2007).

In addition, we cannot rule out that activity in neighboring regions to the targeted DLPFC might also have been modulated by tDCS and contributed to the behavioral changes. The low spatial resolution of tDCS is an inherent limitation of this noninvasive brain stimulation technique. Thus, we cannot rule out the possibility that tDCS of the DLPFC might have influenced other frontal regions such as the orbitofrontal/ventromedial cortex, especially considering that they are densely interconnected (Ghashghaei and Barbas, 2002). However, tDCS over the motor area corresponding to the first dorsal interosseous changes corticospinal excitability for this muscle but not for the abductor digiti minimi or flexor carpi ulnaris, despite the close proximity of their motor cortical representation (Uy and Ridding, 2003), and moving the stimulation electrode 1 or $2 \mathrm{~cm}$ in the anterior direction abolishes this effect (Nitsche et al., 2003a). Future studies including neuroimaging measures to investigate changes in cortical excitability associated with risk-taking behaviors during neuromodulation might shed valuable light onto these issues.

We cannot completely rule out that other variables than the stimulation condition might have influenced the lateralized effect of the DLPFC observed in the present study, such as gender differences. The greater number of women could have contributed to the functional hemispheric asymmetries, because cortical activation depends on the estrogen concentration that differs in relation to the menstrual cycle (Dietrich et al., 2001; Hausmann, 2005; Holländer et al., 2005; Hausmann et al., 2006). However, we believe this is unlikely. Although the number of women and men was unequal, $p$ values for gender differences were far from being statistically significant ( $p=0.46$ for the choice measure; $p=0.69$ for the decision-time measure). Moreover, previous work including only men as participants showed a similar lateralized effect (Knoch et al., 2006a). Handedness might also have contributed to the hemispheric asymmetry observed in the present work. Although we mainly included right-handed volunteers (33 of 36 participants), we did not include cognitive tests to evaluate handedness effects or assess hemispheric dominance and assess (e.g., whether the hemispheric asymmetry for the risk task is correlated with language laterality and mood laterality).

\section{Clinical implications}

Findings from the present study clearly show the feasibility of decreasing risk taking in humans. It is tempting to speculate on potential beneficial therapeutic effects of such an intervention in some clinical populations. Individuals with substance addiction display abnormally risky decision-making behaviors (Grant et al.,
2000; Bechara et al., 2001, 2002; Petry, 2001; Cavedini et al., 2002; Epstein et al., 2006), and their stimulus-bound behaviors often result in real-life negative consequences. Impaired decisionmaking behaviors have also been reported in obese patients (Pignatti et al., 2006) and appear correlated to body mass index (Davis et al., 2004). Neuromodulation might hold promising therapeutic value for such populations. Recent preliminary studies have shown beneficial effects of neuromodulation in such populations. For example, Eichhammer et al. (2003) showed that rTMS to the left DLPFC can decrease the number of cigarettes smoked in nicotine addicts. More recently, reduced craving in smokers was observed after tDCS over both DLPFCs (Fregni et al., 2007). Camprodon et al. (2007) found that high-frequency rTMS to the right (but not the left) DLPFC reduces craving in cocaine addicts. Also, tDCS over both DLPFCs resulted in decreased craving for patients with alcohol dependence (Boggio et al., 2007). Diminished food craving has also been observed in individuals with food craving after one session of high-frequency rTMS over the left DLPFC (Uher et al., 2005). Other populations displaying detrimental decision-making behaviors or abnormal response to reward, such as pathological gamblers (Cavedini et al., 2002; Brand et al., 2005; Goudriaan et al., 2006), patients with bulimia, or those with anorexia nervosa (Davis and Woodside, 2002), might similarly benefit from neuromodulation-based approaches. The present findings provide additional evidence to support and encourage such investigations.

\section{References}

Ardolino G, Bossi B, Barbieri S, Prori A (2005) Non-synaptic mechanisms underlie the after-effects of cathodal transcutaneous direct current stimulation of the human brain. J Physiol (Lond) 568:653-663.

Bechara A, Dolan S, Denburg N, Hindes A, Anderson SW, Nathan PE (2001) Decision-making deficits, linked to a dysfunctional ventromedial prefrontal cortex, revealed in alcohol and stimulant abusers. Neuropsychologia 39:376-389.

Bechara A, Tranel D, Damasio AR (2002) The somatic marker hypothesis and decision-making. In: Handbook of neuropsychology: frontal lobes, Vol 7, Ed 2 (Boller F, Grafman J, eds), pp 117-143. Amsterdam: Elsevier.

Bechara A, Damasio H, Tranel D, Damasio AR (2005) The Iowa Gambling Task and the somatic marker hypothesis: some questions and answers. Trends Cogn Sci 9:159-162.

Boggio PS, Sultani N, Fecteau S, Merabet L, Mecca T, Pascual-Leone A, Basaglia A, Fregni F (2007) Prefrontal cortex modulation using transcranial DC stimulation reduces alcohol craving: a double-blind, sham-controlled study. Drugs Alcohol Depend, in press.

Brand M, Kalbe E, Labudda K, Fujiwara E, Kessler J, Markowitsch HJ (2005) Decision-making impairments in patients with pathological gambling. Psychiatry Res 133:91-99.

Camprodon JA, Martinez-Raga J, Alonso-Alonso M, Shih MC, PascualLeone A (2007) One session of high frequency repetitive transcranial magnetic stimulation (rTMS) to the right prefrontal cortex transiently reduces cocaine craving. Drug Alcohol Depend 86:91-94.

Cavedini P, Riboldi G, Keller R, D’Annuci A, Bellodi L (2002) Frontal lobe dysfunction in pathological gambling patients. Biol Psychiatry 51:334-341.

Davis C, Woodside DB (2002) Sensitivity to the rewarding effects of food and exercise in the eating disorders. Compr Psychiatry 43:189-194.

Davis C, Levitan RD, Muglia P, Bewell C, Kennedy JL (2004) Decisionmaking deficits and overeating: a risk model for obesity. Obes Res 12:929-935.

Dietrich T, Krings T, Neulen J, Willmes K, Erberich S, Thron A, Sturm W (2001) Effects of blood estrogen level on cortical activation patterns during cognitive activation as measured by functional MRI. NeuroImage 13:425-432.

Eichhammer P, Johann M, Kharraz A, Binder H, Pittrow D, Wodarz N, Hajak G (2003) High-frequency repetitive transcranial magnetic stimulation decreases cigarette smoking. J Clin Psychiatry 64:951-953.

Epstein JA, Bang H, Botvin GJ (2006) Which psychosocial factors moderate 
or directly affect substance use among inner-city adolescents? Addict Behav 32:700-713.

Fecteau S, Pascual-Leone A, Zald D, Liguori P, Théoret H, Boggio PS, Fregni F (2007) Activation of prefrontal cortex by transcranial direct current stimulation reduces appetite for risk during ambiguous decision making. J Neurosci 27:6212-6218.

Fregni F, Boggio PS, Nitsche M, Bermpohl F, Antal A, Feredoes E, Marcolin MA, Rigonatti SP, Silva MTA, Paulus W, Pascual-Leone A (2005) Anodal transcranial direct current stimulation of prefrontal cortex enhances working memory. Exp Brain Res 166:23-30.

Fregni F, Boggio PS, Santos MC, Lima M, Vieira AL, Rigonatti SP, Silva MT, Barbosa ER, Nitsche MA, Pascual-Leone A (2006) Noninvasive cortical stimulation with transcranial direct current stimulation in Parkinson's disease. Mov Disord 21:1693-1702.

Fregni F, Liguori P, Fecteau S, Nitsche MA, Pascual-Leone A, Boggio PS (2007) Cortical stimulation of the prefrontal cortex with transcranial DC stimulation to reduce smoking cue-provoked craving: a randomized, sham-controlled study. J Clin Psychiatry, in press.

Gandiga PC, Hummel FC, Cohen LG (2006) Transcranial DC stimulation (tDCS) A tool for double-blind sham-controlled clinical studies in brain stimulation. Clin Neurophysiol 117:845-850.

Gangitano M, Valero-Cabré A, Tormos JM, Mottaghy FM, Romero JR, Pascual-Leone A (2002) Modulation of input-output curves by low and high frequency repetitive transcranial magnetic stimulation of the motor cortex. Clin Neurophysiol 113:1249-1257.

Ghashghaei HT, Barbas H (2002) Pathways for emotion: interactions of prefrontal and anterior temporal pathways in the amygdala of the rhesus monkey. Neuroscience 115:1261-1279.

Goudriaan AE, Oosterlaan J, de Beurs E, van den Brink W (2006) Psychophysiological determinants and concomitants of deficient decision making in pathological gamblers. Drug Alcohol Depend 84:231-239.

Grant S, Contoreggi C, London ED (2000) Drug abusers show impaired performance in a laboratory test of decision making. Neuropsychologia $38: 1180-1187$.

Hausmann M (2005) Hemispheric asymmetry in spatial attention across the menstrual cycle. Neuropsychologia 43:1559-1567.

Hausmann M, Tegenthoff M, Sänger J, Janssen F, Güntürkün O, Schwenkreis P (2006) Transcallosal inhibition across the menstrual cycle: a TMS study. Clin Neurophysiol 117:26-32.

Holländer A, Hausmann M, Hamm JP, Corballis MC (2005) Sex hormonal modulation of hemispheric asymmetries in the attentional blink. J Int Neuropsychol Soc 11:263-272.

Hummel F, Celnik P, Giraux P, Floel A, Wu WH, Gerloff C, Cohen LG (2005) Effects of non-invasive cortical stimulation on skilled motor function in chronic stroke. Brain 128:490-499.

Iyer MB, Mattu U, Grafman J, Lomarev M, Sato S, Wassermann EM (2005) Safety and cognitive effect of frontal DC brain polarization in healthy individuals. Neurology 64:872-875.

Knoch D, Gianotti LR, Pascual-Leone A, Treyer V, Regard M, Hohmann M, Brugger P (2006a) Disruption of right prefrontal cortex by lowfrequency repetitive transcranial magnetic stimulation induces risktaking behavior. J Neurosci 26:6469-6472.

Knoch D, Pascual-Leone A, Meyer K, Treyer V, Fehr E (2006b) Diminishing reciprocal fairness by disrupting the right prefrontal cortex. Science 314:829-832.

Krain AL, Wilson AM, Arbuckle R, Castellanos FX, Milham MP (2006) Dis- tinct neural mechanisms of risk and ambiguity: a meta-analysis of decision-making. NeuroImage 32:477-484.

Liebetanz D, Nitsche MA, Tergau F, Paulus W (2002) Pharmacological approach to the mechanisms of transcranial DC-stimulation-induced after effects of human motor cortex excitability. Brain 125:2238-2247.

Nitsche MA, Paulus W (2000) Excitability changes induced in the human motor cortex by weak transcranial direct current stimulation. J Physiol (Lond) 527:633-639.

Nitsche MA, Paulus W (2001) Sustained excitability elevations induced by transcranial DC motor cortex stimulation in humans. Neurology 57:1899-1901.

Nitsche MA, Liebetanz D, Antal A, Lang N, Tergau F, Paulus W (2003a) Modulation of cortical excitability by weak direct current stimulation technical, safety and functional aspects. Suppl Clin Neurophysiol 56:255-276.

Nitsche MA, Schauenburg A, Lang N, Liebetanz D, Exner C, Paulus W, Tergau F (2003b) Facilitation of implicit motor learning by weak transcranial direct current stimulation of the primary motor cortex in the human. J Cogn Neurosci 15:619-626.

Nitsche MA, Doemkes S, Karakoses T, Antal A, Liebetanz D, Lang N, Tergau F, Paulus W (2007) Shaping the effects of transcranial direct current stimulation of the human motor cortex. J Neurophysiol 97:3109-3117.

Petry NM (2001) Substance abuse, pathological gambling, and impulsiveness. Drug Alcohol Depend 63:29-38.

Pignatti R, Bertella L, Albani G, Mauro A, Molinari E, Semenza C (2006) Decision-making in obesity: a study using the Gambling Task. Eat Weight Disord 11:126-132.

Priori A (2003) Brain polarization in humans: a reappraisal of an old tool for prolonged non-invasive modulation of brain excitability. Clin Neurophysiol 114:589-595.

Purpura D, McMurtry J (1965) Intracellular activities and evoked potential changes during polarization of motor cortex. J Neurophysiol 28:166-185.

Rogers RD, Owen AM, Middleton HC, Williams EJ, Pickard JD, Sahakian BJ, Robins TW (1999) Choosing between small, likely rewards and large, unlikely rewards activates inferior and orbital prefrontal cortex. J Neurosci 20:9029-9038.

Romero JR, Anschel D, Sparing R, Gangitano M, Pascual-Leone A (2002) Subthreshold low frequency repetitive transcranial magnetic stimulation selectively decreases facilitation in the motor cortex. Clin Neurophysiol 113:101-107.

Starkstein SE, Robinson RG (1997) Mechanism of disinhibition after brain lesions. J Nerv Ment Dis 185:108-114.

Uher R, Yoganathan D, Mogg A, Eranti SV, Treasure J, Campbell IC, McLoughlin DM, Schmidt U (2005) Effect of left prefrontal repetitive transcranial magnetic stimulation on food craving. Biol Psychiatry $58: 840-842$.

Uy J, Ridding MC (2003) Increased cortical excitability induced by transcranial DC and peripheral nerve stimulation. J Neurosci Methods 127:193-197.

van't Wout M, Kahn RS, Sanfey AG, Aleman A (2005) Repetitive transcranial magnetic stimulation over the right dorsolateral prefrontal cortex affects strategic decision-making. NeuroReport 16:1849-1852.

Vines BW, Nair DG, Schlaug G (2006) Testing for causality with transcranial direct current stimulation: pitch memory and the left supramarginal gyrus. NeuroReport 17:1047-1050.

Wagner T, Valero-Cabré A, Pascual-Leone A (2007) Noninvasive human brain stimulation. Annu Rev Biomed Eng 9:527-565. 